\title{
LABORATORIO DE REPRODUCCIÓN Y METABOLISMO CEFYBO-CONICET. FACULTAD DE MEDICINA, UBA
}

Directora: Dra. Alicia Jawerbaum

\section{LABORATORY OF REPRODUCTION AND METABOLISM CEFYBO-CONICET. FACULTYY OF MEDICINE, UBA}

Director: Dr. Alicia Jawerbaum

En la gestación las complicaciones inducidas por la diabetes pregestacional y gestacional son múltiples ${ }^{1,2}$. Abarcan alteraciones que pueden afectar el embrión temprano y conducir a fallas en la implantación, anomalías en los procesos de organogénesis temprana que incrementan el riesgo de malformaciones congénitas y fallas en los procesos de desarrollo placentario que pueden vincularse tanto a la mayor inducción de preeclampsia como a anomalías de crecimiento intrauterino (retraso de crecimiento o macrosomía) que afectan el desarrollo perinatal y postnatal. En este contexto son relevantes las alteraciones metabólicas propias de la diabetes materna, tanto del metabolismo de los hidratos de carbono como de los lípidos, y que impactarán en la transferencia de nutrientes al feto y afectarán el desarrollo y crecimiento fetal. Las consecuencias adversas se presentan en la madre (donde a las complicaciones propias de la diabetes se suma el fuerte impacto de los cambios adaptativos propios de la gestación), en el desarrollo embrionario, placentario y fetal, en las complicaciones perinatales y en la vida del neonato, en el cual es elevado el riesgo de programación de alteraciones metabólicas y cardiovasculares ${ }^{3,4}$.

En el Laboratorio de Reproducción y Metabolismo, desde hace más de 25 años y a través de la generación y caracterización de diversos modelos experimentales de diabe- tes y preñez, investigamos acerca de los mecanismos de daño inducidos por la diabetes en diferentes etapas de la gestación y en la progenie $^{5}$. Fuimos pioneros en la identificación del rol del estrés oxidativo y nitrativo en anomalías inducidas por la diabetes materna en etapas de implantación, placentación, desarrollo embrionario, fetal y placentario ${ }^{6}$. En forma más reciente pudimos identificar la importancia de estos procesos en la programación del desarrollo de patologías metabólicas y cardíacas en la progenie en modelos experimentales de diabetes y preñez ${ }^{7}$. En forma paralela, realizamos estudios en pacientes donde identificamos el mismo tipo de anomalías pro-oxidantes y pro-inflamatorias y su vinculación con los adversos procesos de remodelación de la matriz extracelular en la placenta a término de pacientes con diabetes ${ }^{3,8}$.

Hacia el año 2000, en la búsqueda de mecanismos endógenos capaces de reducir el adverso entorno pro-oxidante y pro-inflamatorio intrauterino, comenzamos a centrarnos en el estudio de los receptores activados por proliferadores peroxisomales o PPARs ${ }^{9}$. El resultado fue la identificación de importantes procesos de desarrollo regulados por estos receptores nucleares y una clara desregulación de estos factores de transcripción activados por ligandos de naturaleza lipídica evidente a nivel intrauterino en la diabetes materna ${ }^{10}$. Entre los 
hallazgos se destacan la relevancia de anomalías en los niveles y acción de PPARdelta en procesos de dismorfogénesis embrionaria inducida por la diabetes materna, la importante acción antiinflamatoria de PPARgamma a nivel placentario y la acción de PPARalfa vinculada a la regulación del metabolismo lipídico y el crecimiento fetal ${ }^{11-13}$. La función antiinflamatoria y metabólica de los PPAR en la placenta y la reducción en los niveles de PPARgamma y PPARalfa placentarios inducida por la diabetes materna fueron identificadas tanto en modelos animales como en pacientes con diabetes en la gestación ${ }^{14}$.

Teniendo en cuenta que los PPAR pueden ser activados por ligandos de naturaleza lipídica que incluyen diversos ácidos grasos insaturados, nuestro próximo paso fue tratar animales con dietas enriquecidas en ácidos grasos insaturados y conocer que los mismos son transportados en forma facilitada a través de la placenta hacia el feto en desarrollo. Hemos comprobado que estas dietas, administradas a animales con diabetes durante la gestación, reducen la reabsorción embrionaria, las malformaciones congénitas, el entorno pro-oxidante/pro-inflamatorio intrauterino y regula aspectos del metabolismo lipídico en diferentes órganos fetales ${ }^{10,15}$. Además nuestros estudios recientes y en progreso evidencian la capacidad de dichos tratamientos dietarios de mejorar parámetros pro-oxidantes y pro-inflamatorios y regular el metabolismo lipídico en la cría adulta de estos animales, sugiriendo la capacidad de los mismos de

\section{BIBLIOGRAFÍA}

1. McCance DR. Diabetes in pregnancy. Best Pract Res Clin Obstet Gynaecol 2015; 29:685-99.

2. Hod M, Kapur A, Sacks DA, Hadar E, Agarwal M, Di Renzo GC, et al. The International Federation of Gynecology and Obstetrics (FIGO). Initiative on gestational diabetes mellitus: a pragmatic guide for diagnosis, management and care. Int J Gynaecol Obstet 2015; 131 Suppl 3:S173. prevenir, al menos en parte, la adversa programación intrauterina?.

Otros aspectos actuales en estudio se vinculan a la identificación de un nóvel modelo de diabetes gestacional inducido por programación intrauterina ${ }^{16}$ y a las investigaciones realizadas en modelos de sobreingesta de grasas saturadas, que abarcan estudios metabólicos durante la gestación y en la progenie ${ }^{17,18}$.

Asimismo se encuentran en progreso en el laboratorio estudios que vinculan a la vía de Ios PPAR y de mTOR, sabiendo que es poco aún lo que se conoce de las vías que regulan la síntesis proteica y la transferencia de aminoácidos durante el desarrollo embrionario y feto-placentario en la diabetes materna, y que podría explicar mecanismos de aberrante crecimiento y desarrollo intrauterino.

Por último, se encuentran en progreso estudios de regulación epigenética de los PPAR, ya que los mismos podrían explicar las alteraciones intergeneracionales de los PPAR a nivel intrauterino, importantes por su vínculo con el entorno pro-oxidante, pro-inflamatorio y de regulación metabólica durante el desarrollo y en la progenie.

En resumen, el equipo de trabajo del Laboratorio de Reproducción y Metabolismo estudia aspectos metabólicos y su vinculación con procesos de señalización intracelular y la acción de factores de transcripción relevantes en el desarrollo embrionario, placentario y fetal, y cuyas alteraciones inducidas por la diabetes materna tienen repercusión a nivel gestacional y postnatal.
3. Lappas M, Hiden U, Desoye G, Froehlich J, Hauguel-de Mouzon S, Jawerbaum A. The role of oxidative stress in the pathophysiology of gestational diabetes mellitus. Antioxid Redox Signal 2011; 15:3061-100.

4. Mitanchez D, Yzydorczyk C, Simeoni U. What neonatal complications should the pediatrician be aware of in case of maternal gestational diabetes? World J Diabetes $2015 ; 6: 734-43$. 
5. Jawerbaum A, White V. Animal models in diabetes and pregnancy. Endocr Rev 2010; 31:680-701.

6. Jawerbaum A, González E. Diabetic pregnancies: the challenge of developing in a pro-inflammatory environment. Curr Med Chem 2006; 13:2127-38.

7. Capobianco E, Pelesson M, Careaga V, Fornes D, Canosa I, Higa R, et al. Intrauterine programming of lipid metabolic alterations in the heart of the offspring of diabetic rats is prevented by maternal diets enriched in olive oil. Mol Nutr Food Res 2015; 59:1997-2007.

8. Capobianco E, White V, Sosa M, Marco ID, Basualdo MN, Faingold $\mathrm{MC}$, et al. Regulation of matrix metalloproteinases 2 and 9 activities by peroxynitrites in term placentas from type 2 diabetic patients. Reprod Sci 2012; 19:814-22.

9. Wahli W, Michalik L. PPARs at the crossroads of lipid signaling and inflammation. Trends Endocrinol Metab 2012; 23:351-63.

10. Jawerbaum A, Capobianco E. Review: effects of PPAR activation in the placenta and the fetus: implications in maternal diabetes. Placenta 2011; 32 Suppl 2:S212-7.

11. Higa R, González E, Pustovrh MC, White V, Capobianco E, Martínez N, et al. PPARdelta and its activator PGI2 are reduced in diabetic embryopathy: involvement of PPARdelta activation in lipid metabolic and signalling pathways in rat embryo early organogenesis. Mol Hum Reprod 2007; 13:103-10.

12. Pustovrh MC, Capobianco E, Martínez N, Higa R, White $\mathrm{V}$, Jawerbaum A. MMP/TIMP balance is modulated in vitro by $15 \mathrm{dPGJ}(2)$ in fetuses and placentas from diabetic rats. Eur J Clin Invest 2009; 39:1082-90.
13. Martínez N, Capobianco E, White V, Pustovrh MC, Higa $R$, Jawerbaum A. Peroxisome proliferator-activated receptor alpha activation regulates lipid metabolism in the feto-placental unit from diabetic rats. Reproduction 2008; 136:95-103.

14. Capobianco E, Martínez N, Fornes D, Higa R, Di Marco I, Basualdo MN, et al. PPAR activation as a regulator of lipid metabolism, nitric oxide production and lipid peroxidation in the placenta from type 2 diabetic patients. Mol Cell Endocrinol 2013; 377:7-15.

15. Kurtz M, Capobianco E, Martínez N, Roberti SL, Arany E, Jawerbaum A. PPAR ligands improve impaired metabolic pathways in fetal hearts of diabetic rats. J Mol Endocrinol 2014; 53:237-46.

16. Capobianco E, Fornes D, Linenberg I, Powell TL, Jansson $\mathrm{T}$, Jawerbaum A. A novel rat model of gestational diabetes induced by intrauterine programming is associated with alterations in placental signaling and fetal overgrowth. Mol Cell Endocrinol 2016; 422:221-32.

17. Mazzucco MB, Fornes D, Capobianco E, Higa R, Jawerbaum A, White V. Maternal saturated-fat-rich diet promotes leptin resistance in fetal liver lipid catabolism and programs lipid homeostasis impairments in the liver of rat offspring. J Nutr Biochem 2016; 27:61-9.

18. Jawerbaum A, White V. Review on intrauterine programming: consequences in rodent models of mild diabetes and mild fat overfeeding are not mild. Placenta 2017; 52:21-32. 